\section{Review: early intervention services can be clinically beneficial for people with early psychosis}

\section{QUESTION}

Question: How effective are early intervention services, cognitive behavioural therapy (CBT) and family interventions for people with early psychosis?

Outcomes: Duration of untreated psychosis, hospital admissions, psychotic relapse, mean positive and negative symptoms (measurement scales: Positive and Negative Syndrome Scale, Scale for Assessment of Positive Symptoms, Scale for Assessment of Negative Symptoms, Brief Psychiatric Rating Scale).

\section{METHODS}

Design: Systematic review and meta-analysis.

Data sources: CINAHL, CENTRAL, EMBASE, MEDLINE and PsycINFO searched from database inception to September 2009; some hand searching and backwards citation checking.

Study selection and analysis: Inclusion criteria: Patients with diagnosis of early psychosis (clinical diagnosis within 5 years of first presentation or episode). Exclusion criteria: Interventions targeted at prepsychotic or prodromal patients, or those in high-risk groups; trials that did not clearly report randomisation; trials with fewer than 10 participants. The Scottish Intercollegiate Guidelines Network checklist was used to assess eligibility. Two authors extracted study details, and three extracted outcome data. One author checked for accuracy.

\section{MAIN RESULTS}

Four trials ( $\mathrm{n}=800$ ) were included in meta-analysis of the effects of early intervention services. Early intervention services reduced likelihood of relapse compared with standard care (2 randomised controlled trials (RCTs), RR $0.66,95 \%$ CI 0.47 to 0.94 ) and reduced hospital admissions (3 RCTs, RR 0.67, 95\% CI 0.54 to 0.83 ). Psychosis symptoms were also reduced (positive symptoms: 2 RCTs, standardised mean difference (SMD) $-0.21,95 \%$ CI -0.42 to -0.01 ; negative symptoms: 2 RCTs, SMD $-0.39,95 \%$ CI -0.57 to -0.20 ).

Three trials $(\mathrm{n}=288$ ) of family interventions were identified. The intervention reduced risk of relapse and hospital admission combined compared with standard care (composite RR $0.50,95 \%$ CI 0.32 to 0.80 ), but this effect was not observed with relapse and hospital admission as single end points.

Four trials $(n=620)$ of CBT-based interventions found a reduction in positive symptoms compared with usual care after 2-year post-treatment follow-up (SMD -0.60, 95\% CI -0.79 to -0.41 ) but not at end of treatment. Negative symptoms also showed an improvement at 2-year follow-up only (SMD $-0.45,95 \%$ CI -0.80 to -0.09 ). There was no difference in rates of hospital admission or relapse between patients undergoing $\mathrm{CBT}$ and those receiving usual care.

\section{CONCLUSIONS}

Early intervention services, family intervention and CBT all showed some benefits for treatment for early psychosis when compared with standard care. Early intervention services seem to give the widest range of benefits by improving symptoms and reducing risk of hospital admission and relapse. These effects were observable by the end of treatment.

\section{ABSTRACTED FROM}

Bird V, Premkumar P, Kendall T, et al. Early intervention services, cognitive-behavioural therapy and family intervention in early psychosis: systematic review. Br J Psychiatry 2010;197:350-6

Correspondence to: V Bird, National Collaborating Centre for Mental Health, Royal College of Psychiatrists' Research and Training Unit, Standon House, 21 Mansell Street, London E1 8AA, UK; vbird@cru.rcpsych.ac.uk

Source of funding: NICE, National Collaborating Centre for Mental Health.
E rly intervention services have developed over the last two decades ${ }^{1}$ going from services that were largely research based to becoming wellfunded parts of some countries' mental health systems. Many of these services have developed from a common model that provides an enhanced range of psychological and psychosocial services in conjunction with low-dose antipsychotic medication to people with a first episode of psychosis, with intensive and sustained treatment usually offered over periods ranging from 18 months to 5 years. Although there is now some evidence that early intervention is cheaper ${ }^{2}$ and makes a positive difference in terms of short and longer term outcomes, little is known about the relative contribution or importance of subcomponents of early intervention services. In order to refine and improve on the early intervention model, it will be increasingly important to examine these subcomponents.

The study by Bird and colleagues finds that there are benefits to early intervention as an approach to treating early psychosis and that there are specific benefits of cognitive behavioural therapy (CBT) and family therapy on symptoms and relapse, respectively. More interestingly, the benefits of CBT are not immediately apparent but become manifest over a 2-year follow-up period. The results of this study have implications for service provision and practice as well as for policy makers in a time of potential reduced funding for mental health services.

In regards to service provision, these results would suggest that the provision of early treatment by itself is good but not enough. Further benefits can be gained through the addition of specific therapies to address specific problems. Such therapies as CBT and family therapy can enhance the benefit of early recognition, reduced duration of untreated psychosis and good pharmacological treatment. Likewise, there is increasing recognition that other psychosocial interventions, specifically targeted, can also enhance recovery. ${ }^{3}$

For funders and policy makers, although there may be pressure to reduce the complex mix of services to early intervention services, this would actually result in only a short-term financial benefit. The evidence shows that the long-term benefits of reduced symptoms (through CBT) and reduced relapse and rehospitalisation (through family therapy) include greater levels of employment and social inclusion and subsequent reduced burden on welfare and services.

\section{Eóin Killackey \\ Centre for Youth Mental Health, The University of Melbourne, Melbourne, Victoria, Australia}

Competing interests EK has received speaker fees from Janssen Cilag and Eli Lilly for speaking on psychosocial interventions in psychosis. He has also received research funding from Bristol Myers Squibb.

\section{REFERENCES}

1. Killackey $\mathbf{E}$, Yung AR. Effectiveness of early intervention in psychosis. Curr Opin Psychiatry 2007:20:121-5.

2. Mihalopoulos C, Harris M, Henry L, et al. Is early intervention in psychosis cost-effective over the long term? Schizophr Bull 2009;35:909-18.

3. Rinaldi M, Killackey E, Smith J, et al. First episode psychosis and employment: a review. Int Rev Psychiatry 2010;22:148-62. 